\title{
Potential Benefits from the Use of the Supraclavicular Artery Island Flap for Immediate Soft-Tissue Reconstruction During the COVID-19 Pandemic
}

\author{
Alexandra Thompson ${ }^{1}$ (D) Zia Khan ${ }^{1} \cdot$ Alan Patterson $^{1} \cdot$ Kapil Java $^{1} \cdot$ \\ Iain Varley ${ }^{1}$
}

Received: 30 May 2020/Accepted: 18 August 2020/Published online: 28 August 2020

(C) The Association of Oral and Maxillofacial Surgeons of India 2020

\begin{abstract}
Introduction The coronavirus disease 19 (COVID-19) pandemic has placed significant strains on healthcare resources including staff, theatre, high dependency and intensive care availability. Surgery remains the optimal treatment for the majority of oral malignancies, and primary reconstruction is often necessary to avoid significant morbidity as a result of ablative procedures.

Description The supraclavicular artery island flap (SCAIF) is increasingly finding a place as an alternative to free flap reconstruction of soft-tissue defects and has several specific advantages when compared to use of a soft-tissue free flap during the COVID-19 pandemic. It can be used for a range of head and neck defects.

Results We describe our experience with the SCAIF during the pandemic in five patients with a variety of tumour types and locations. All five patients had a successful outcome with none requiring further reconstruction and all had a functional swallow postoperatively, including a patient who underwent a total pharyngeal reconstruction with a SCAIF.
\end{abstract}

Alexandra Thompson

alexandra.thompson6@nhs.net

Zia Khan

Zia.Khan@nhs.net

Alan Patterson

Alan.patterson1@nhs.net

Kapil Java

Kapil.Java@nhs.net

Iain Varley

Iainvarley@gmail.com

1 Sheffield Teaching Hospitals, Royal Hallamshire Hospital, Glossop Road, Sheffield S10 2JF, England
Discussion The use of a SCAIF during the current COVID-19 pandemic has significant advantages and is a reliable alternative to a soft tissue free flap.

Keywords COVID-19 - Reconstruction · Supraclavicular artery island flap · Oncology

\section{Introduction}

On 17 March 2020, the British Association of Head and Neck Oncologists published guidelines regarding head and neck oncology management during the COVID-19 pandemic [1]. These recommend that operative time, surgical personnel and use of intensive care resources should all be minimised where possible. This leaves the head and neck surgeon with a clear dilemma: resection of head and neck malignancy and optimal functional reconstruction is resource intensive, requiring long operating times, high numbers of theatre and surgical staff and high-dependency post-operative care. Microvascular reconstruction with free tissue transfer is also challenging while using recommended personal protective equipment (PPE) [2]. The postoperative course may be complicated by return to theatre for flap salvage or flap failure and secondary reconstruction. There is a requirement for post-operative respiratory or inotropic support, and an appreciable rate of medical complications such as hospital-acquired pneumonia [3]. If primary reconstruction is avoided, in order to minimise the duration of the initial surgery then any advantage gained may be eroded by morbidity such as inability to regain functional swallowing, potentially resulting in increased length of stay, need for additional procedures (such as gastrostomy) and need for delayed reconstruction, resulting 
in higher pressure on resources once the pandemic has passed.

Prior to the COVID-19 pandemic, we had found the supraclavicular artery island flap (SCAIF) useful as a reconstructive option for selected patients in whom microvascular tissue transfer was undesirable. The SCAIF is a thin, pliable, axially-based flap, with similar handling characteristics to the radial forearm free flap (RFFF). We have used it for a variety of defects, from total pharyngeal reconstruction, for resections throughout the oral cavity from floor of mouth to tongue, buccal mucosa and palate, and for skin defects. It is pedicled, reliable, straightforward to raise and has low donor-site morbidity [4]. Giving due consideration to reducing length of operating time and reducing the number of personnel in theatre during the COVID-19 pandemic, we are avoiding elective neck dissection and reconstruction where possible, but in those patients where immediate reconstruction of soft tissues is considered unavoidable, we are now routinely using the SCAIF in preference to a free flap.

\section{Methods}

We preferentially use the flap in association with a level I-IV selective neck dissection. Care must be taken when dissecting level IV to preserve the transverse cervical vessels as the supraclavicular artery usually arises from the transverse cervical artery in level V. In patients who have had previous neck surgery, or where a radical neck dissection is required, location of the artery can be confirmed over the clavicle using a hand-held Doppler probe. Care must be taken to angle the probe so as to avoid picking up subclavian vessels in error. The desired skin flap is marked over deltoid, and a broad pedicle marked to the clavicle (Fig. 1).

Initial dissection is straightforward dissection and begins distally down to muscle. The skin incision is completed, and the flap is elevated from distal to proximal in a subfascial plane. The cephalic vein may be encountered anteriorly at the deltopectoral groove, and this can be dissected free from the flap and preserved.

The proximal flap is de-epithelised, and the fat and fascia at the anterior and posterior borders of the desired pedicle incised as the dissection continues to clavicle.

The artery may be located at this point by either transillumination or by Doppler probe (Fig. 2).

Dissection over the clavicle must be undertaken with care. The periosteum is incised on the anterior border of the clavicle (often a deep perforator is encountered here that must be cauterised), and the periosteum is stripped over the clavicle, incising the lateral and medial edges. The periosteum is then incised at the posterior border to allow the flap to elevate fully - care must be taken at this point as the artery lies just above the periosteum, but the incision must be completed to allow full mobility of the flap. The viability of the flap at this point can be confirmed by assessing capillary refill of the skin paddle.

Dissection into level V crosses tissue planes-the neck incision is in a subplatysmal plane while the flap lies superficial laterally, incorporating the fascia that becomes contiguous with platysma as it progresses proximally and then crossing into the deep neck at level V. We have found it helpful to approach the dissection at this point from the

Fig. 1 Skin flap markings

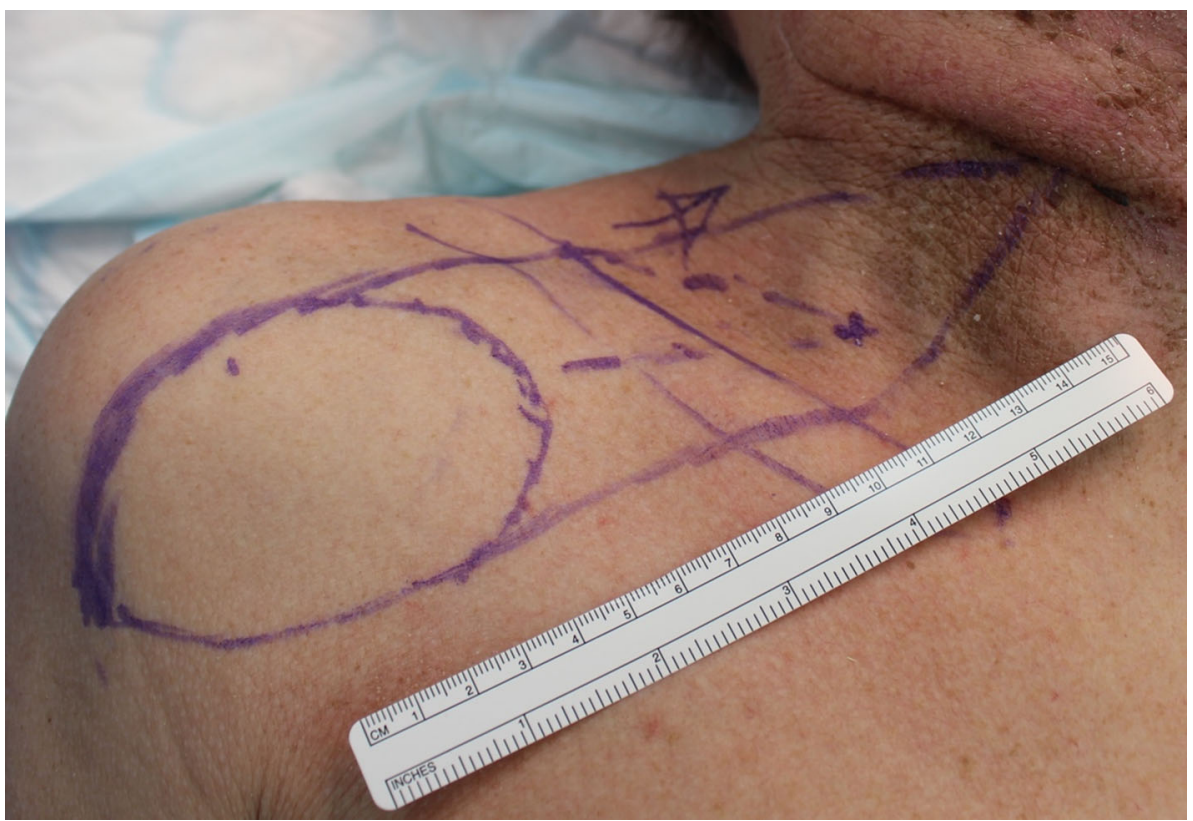


Fig. 2 Confirmation of the pedicle vessel with intraoperative Doppler and transillumination but is not always readily identifiable

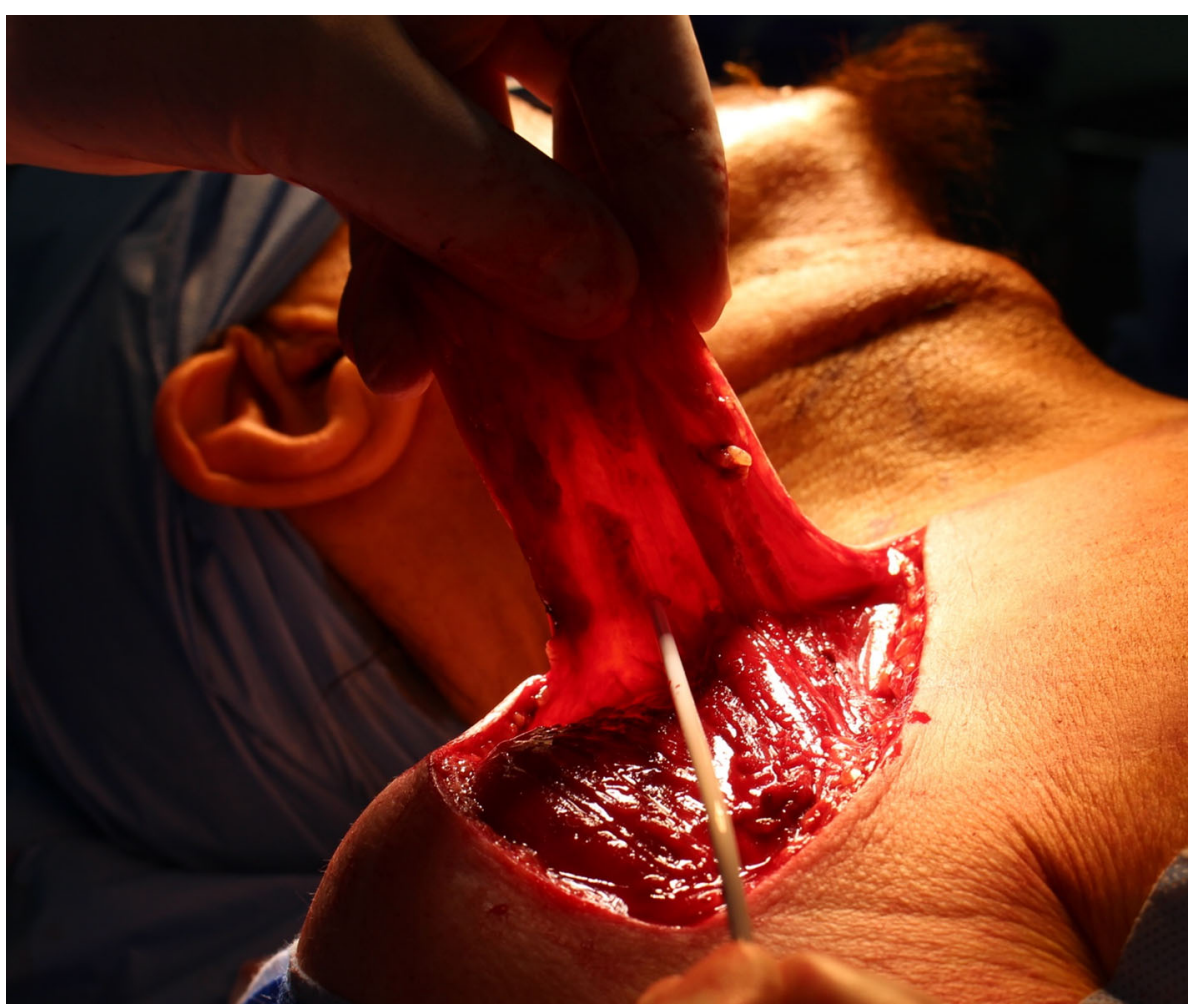

neck, dissecting around the posterior border of sternomastoid to skeletonise the muscle. This allows the flap to be turned over and passed deep to sternomastoid in order to increase the reach to the oral cavity, where it can be inset.

Wide undermining of the chest anteriorly and over trapezius posteriorly allows primary closure over a suction drain.

Fig. 3 Patient 1 -SCC right posterolateral tongue

\section{Results}

In the first 4 week of the COVID-19 pandemic, we have performed 17 oncological resections for oral cavity cancer including 4 where we have used a SCAIF as primary reconstruction (Figs. 3,4) in patients who, in our unit prior to the pandemic, would have undergone primary reconstruction with a soft-tissue free flap (Table 1).

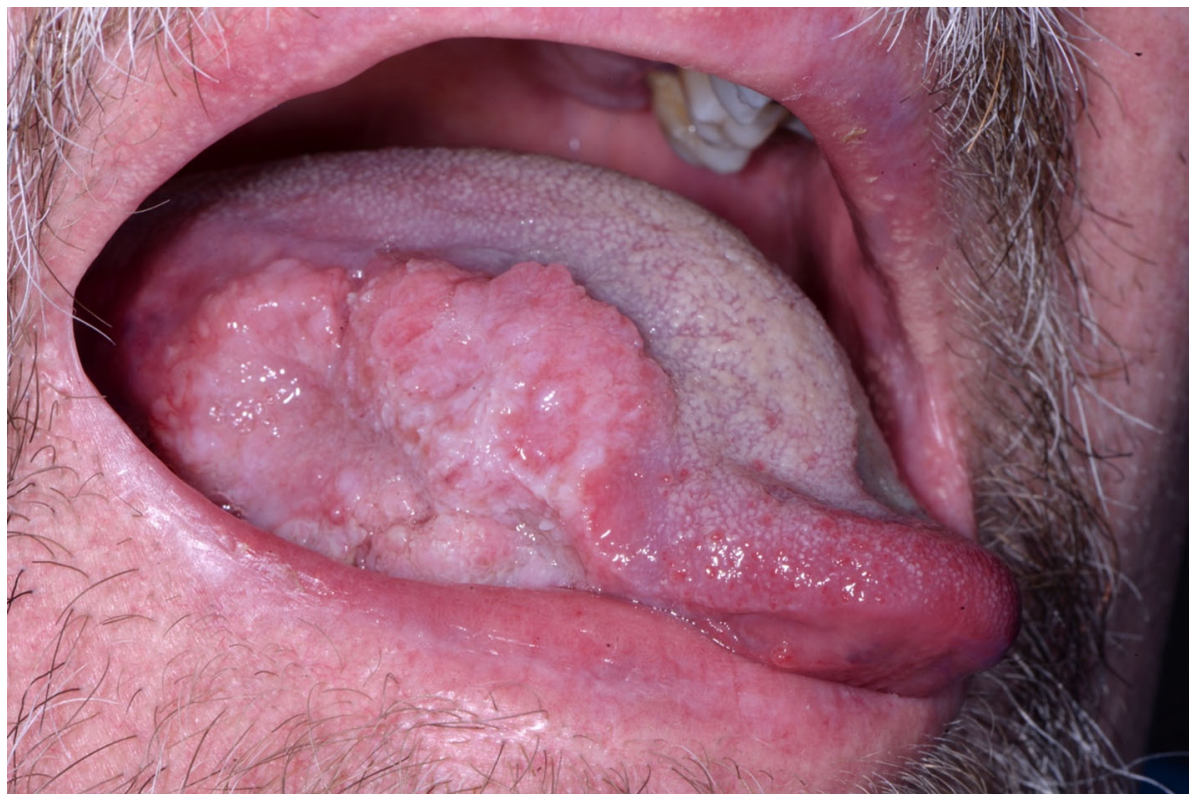


Fig. 4 Patient 1 at three weeks post op, intra-oral view

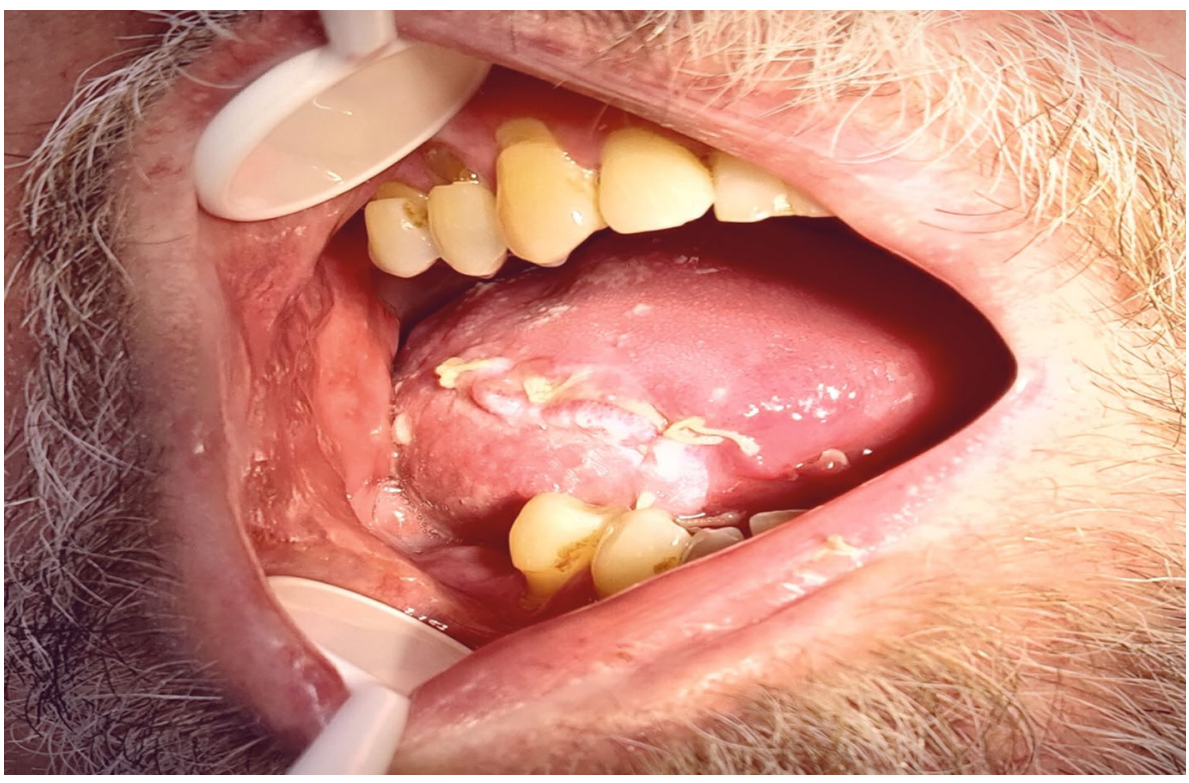

Table 1 Patient characteristics

\begin{tabular}{|c|c|c|c|c|c|}
\hline Patient & Age/sex & Tumour site & $\begin{array}{l}\text { Tumour stage } \\
\text { (TNM 8) }\end{array}$ & $\begin{array}{l}\text { ACE-27/ } \\
\text { WHO PS }\end{array}$ & Procedure \\
\hline 1 & $61 \mathrm{M}$ & $\begin{array}{l}\text { SCC lateral } \\
\text { tongue }\end{array}$ & $\mathrm{T} 3 \mathrm{~N} 3 \mathrm{bM} 0$ & $1 / 0$ & $\begin{array}{l}\text { Tracheostomy, right radical neck dissection, resection of right tongue, } \\
\text { SCAIF }\end{array}$ \\
\hline 2 & $49 \mathrm{~F}$ & $\begin{array}{l}\text { SCC Floor of } \\
\text { mouth }\end{array}$ & T2N0M0 & $3 / 1$ & $\begin{array}{l}\text { Tracheostomy, bilateral selective I-IV neck dissections, resection of } \\
\text { floor of mouth, SCAIF }\end{array}$ \\
\hline 3 & $63 \mathrm{M}$ & $\begin{array}{l}\text { SCC floor of } \\
\text { mouth }\end{array}$ & T3N1M0 & $1 / 0$ & $\begin{array}{l}\text { Right selective I-IV neck dissection, resection of right floor of mouth, } \\
\text { SCAIF }\end{array}$ \\
\hline 4 & $42 \mathrm{M}$ & $\begin{array}{l}\text { SCC lateral } \\
\text { tongue }\end{array}$ & T2N1M0 & $1 / 0$ & Left selective I-IV neck dissection, resection of left tongue, SCAIF \\
\hline 5 & $70 \mathrm{M}$ & SCC larynx & T4aN0M0 & $1 / 1$ & $\begin{array}{l}\text { Total laryngopharyngectomy and thryoidectomy, left pectoralis major } \\
\text { flap, right SCAIF }\end{array}$ \\
\hline
\end{tabular}

ACE-27 adult comorbidity evaluation 27. WHO PS World Health Organisation Performance Status

Two patients underwent tracheostomy during the initial surgery, while in two it was avoided. One of these required a tracheostomy post-operatively when they developed a neck haematoma following a post-operative bleed from the lingual artery stump and went on to develop some skin paddle necrosis but required no further reconstruction. A further flap suffered some skin paddle necrosis following development of hospital-acquired pneumonia, again undergoing debridement under local anaesthetic, with no requirement for further reconstruction and required debridement. All patients resumed oral diet with a functional swallow. Of particular note was patient 5 who had a total pharyngeal reconstruction with a SCAIF, the postoperative contrast swallow assessment shows near perfect function with no stricture or leak. Complications and COVID-19 status are summarised below (Table 2).

\section{Discussion}

Interest surrounding the SCAIF as a potential alternative to the soft-tissue RFFF has been increasing in recent years [5]. The handling properties and tissue match are similar to those of a radial forearm free flap, and it reaches the majority of oral defects without exceeding the anatomical constraints of pedicle length. As a thin pliable flap, it has significant advantages over the pectoralis major flap and as the donor site is closed primarily with a favourable aesthetics and function it confers additional benefit over other local flaps, for example the nasolabial flap.

It has several potential benefits at a time when surgical resources are limited. It reduces operative time when compared with free flap reconstruction, with a systematic review showing a reduction in operative time for the 
Table 2 Complications and outcomes

\begin{tabular}{|c|c|c|c|c|}
\hline Patient & Flap complications & Respiratory complications & $\begin{array}{l}\text { CoViD } \\
\text { PCR }\end{array}$ & Other complication \\
\hline 1 & No & No & $\begin{array}{l}- \text { ve } \\
\text { POD3 }\end{array}$ & Constipation \\
\hline 2 & $\begin{array}{l}\text { Some skin paddle necrosis—debrided, no } \\
\text { further reconstruction required }\end{array}$ & Hospital-acquired pneumonia POD2 & $\begin{array}{l}-\mathrm{ve} \\
\text { POD6 }\end{array}$ & No \\
\hline 3 & No & No & $\begin{array}{l}-\mathrm{ve} \\
\text { POD5 }\end{array}$ & $\begin{array}{l}\text { Sinus tachycardia with short runs of } \\
\text { ventricular tachycardia }\end{array}$ \\
\hline 4 & $\begin{array}{l}\text { Some skin paddle necrosis-debridement } \\
\text { under local anaesthetic }\end{array}$ & $\begin{array}{l}\text { Post-operative bleed necessitating } \\
\text { emergency tracheostomy }\end{array}$ & $\begin{array}{l}\text { Not } \\
\text { tested }\end{array}$ & Otitis externa \\
\hline 5 & No & No & $\begin{array}{l}-\mathrm{VE} \\
\text { POD3 }\end{array}$ & No \\
\hline
\end{tabular}

POD post-operative day, CoViD PCR polymerase chain reaction test for COVID-19

SCAIF in comparison with the RFFF [6], both by more rapid flap harvest and by avoiding the need for a microvascular anastomosis. It reduces the number of people required in the operating theatre, at least in our practice-our surgical team within the theatre now consists of 2-3 surgeons, 1 scrub practitioner and 1 Operating Department Assistant (ODA). This limits the number of people exposed to aerosol generation during surgery. Concurrent "two team" operating, as would be our normal practice for a free flap, would require an additional 4 staff members-2 surgeons, a scrub practitioner and an ODA. With an increase in theatre personnel comes an increased consumption of PPE which adds to financial cost and use of what may be a scarce resource. If the flap was not raised by a second team, then the sequential operating for resection and then reconstruction would add significantly to time in theatre. It may reduce the burden on high dependency, with fewer patients requiring ICU and shorter length of stay for those who did require ICU compared with those having RFFF reconstruction [7].

We have had no donor site complications to date, with all sites closed primarily. Our previous experience is that sensation in the flap can be retained in up to $20 \%$ of patients, which is reflected in other published series [8], although this has not occurred in the patients included here. Previous authors have reported an appreciable incidence of salivary fistula with SCAIFs [9], although this has not been our experience, and all patients have returned to oral intake uneventfully.

A meta-analysis of skin loss for SCAIF vs RFFF showed no significant difference, although there was a trend towards partial skin loss in the SCAIF with the retention of underlying fascia which then mucosalises, as compared to a trend towards total flap loss with the RFFF group [6]. This is again representative of our experience with SCAIFs, in this series and also previously where the small number of patients who have had some skin loss have not required secondary reconstruction and have healed uneventfully.

Functionally, the SCAIF is non-inferior to the RFFF, with swallowing as observed by barium swallow similar [7]. Speech quality in comparison with free flap reconstruction does not appear to have been assessed to date.

As the SCAIF is a pedicled flap, the burden on aftercare is also reduced-regular flap observations are not required and the chance of the patient requiring a return to theatre for recipient or donor-site complications post-operatively is also reduced [10]. Our usual practice is to nurse patients in a high-dependency (level 2) bed after a flap reconstruction, but in our experience a patient with a SCAIF can be nursed on a head and neck ward. This reduces the burden on level 2 beds at a time when they are under increased pressure.

\section{Conclusion}

The use of a SCAIF in the current pandemic allows immediate soft-tissue reconstruction with reduced operating time, reduced exposure of theatre staff, reduced requirements for post-operative care and functional results similar to those achieved with RFFF. The SCAIF is straightforward to harvest (with care taken in levels IV and $\mathrm{V}$ of the neck), and the donor site can be closed primarily. We have no hesitation in recommending the SCAIF as a reliable alternative to a soft-tissue free flap during the COVID-19 pandemic.

\section{Acknowledgements None}

Funding This research did not receive any specific grant from funding agencies in the public, commercial or non-for-profit sectors. 


\section{References}

1. BAHNO Statement on Covid-19 BAHNO-British Association of Head \& NeckOncologists. https://www.bahno.org.uk/bahno_ statement_on_covid-19.aspx Accessed 20 Apr 2020

2. Ellis R, Hay-David AGC, Brennan PA (2020) Operating during the COVID-19 pandemic: How to reduce medical error. Br J Oral Maxillofac Surg 58:577-580

3. McMahon J, Handley TPB, Bobinskas A, Elsapagh M, Anwar HS, Ricciardo PV et al (2017) Postoperative complications after head and neck operations that require free tissue transferprevalent, morbid, and costly. $\mathrm{Br} \mathrm{J}$ Oral Maxillofac Surg 1(55):809-814

4. Chiu ES, Liu PH, Friedlander PL (2009) Supraclavicular artery island flap for head and neck oncologic reconstruction: Indications, complications, and outcomes. Plast Reconstr Surg 124:115-123

5. Trautman J, Gore S, Potter M, Clark J, Hyam D, Tan NC et al (2018) Supraclavicular flap repair in the free flap era. ANZ J Surg $88: 540-546$
6. Sukato DC, Timashpolsky A, Ferzli G, Rosenfeld RM, Gordin EA (2019) Systematic review of supraclavicular artery island flap vs free flap in head and neck reconstruction. Otolaryngol Head Neck Surg 160:215-222

7. Welz C, Canis M, Schwenk-Zieger S, Spiegel JL, Weiss BG, Pilavakis Y (2017) Oral cancer reconstruction using the supraclavicular artery island flap: comparison to free radial forearm flap. J Oral Maxillofac Surg 75:2261-2269

8. Atallah S, Guth A, Chabolle F, Bach CA (2015) Supraclavicular artery island flap in head and neck reconstruction. Eur Ann Otorhinolaryngol Head Neck Dis 132:291-294

9. Kokot N, Mazhar K, Reder LS, Peng GL, Sinha UK (2013) The supraclavicular artery island flap in head and neck reconstruction: applications and limitations. JAMA Otolaryngol Head Neck Surg 139:1247-1255

10. UK National Flap Registry I BAPRAS. http://www.bapras.org.uk/ professionals/uk-national-flap-registy Accessed 19 Apr 2020

Publisher's Note Springer Nature remains neutral with regard to jurisdictional claims in published maps and institutional affiliations. 